\title{
Speckle Reduction Using Adaptive Receive-Side Compounding
}

\author{
Are Charles Jensen (1,2), Andreas Austeng (2) \\ (1) Norwegian Computing Center, 0314 Oslo, Norway. \\ (2) Department of Informatics, University of Oslo, 0316 Oslo, Norway.
}

\begin{abstract}
A typical approach to reduce speckle in coherent imaging systems, is to average same-target images with different speckle realizations. We study settings where such realizations come from applying different transducer-array element-weights at reception, referred to here as receive compounding. An effect of such compounding is reduced spatial resolution, causing smearing of point-like image structures, filling of cysts and expansion of hyperechoic regions. In this paper we study how these unwanted effects can be mitigated by combining the compounding with a small, phase-based, adaptive steering of the array at reception. The adaptivity is based on a criterion akin to that of the Capon beamformer; a minimum-output distortionless response. Here, the distortionless part ensures that however we steer, we have a uniform at-focus response. We have applied this adaptive steering in combination with several receive compounding techniques on simulated Field II, phantom and in vivo data. The results show that all the studied compounding techniques respond to this positively in light of the mentioned unwanted effects. The technique based on Thomson's multitaper method even surpassed the non-compounded equivalent in reproducing the geometry of structures. The speckle reduction, as measured by the change in the pixel mean to standard deviation ratio, is indeed lower, and there are subtle changes in the spatial speckle patterns when applying steering; however, we believe that in most cases the negative effects are tolerable in light of the benefits gained. The suggested approach is intuitive and easily implemented.
\end{abstract}

\section{INTRODUCTION}

A characteristic of coherent imaging is the presence of noise-like patterns known as speckle, which stem from constructive and destructive interference. In most medical ultrasound imaging applications, speckle is regarded an artifact of the system that reduces lesion detection and the speckle signal-to-noise level [1, Ch. 8 \& 10]. Large efforts are therefore put into algorithms for speckle reduction. These techniques are often dichotomized into approaches based on obtaining, and then combining, images with different speckle realizations, and post image-formation filtering approaches. The first category comprises observing the target region from different angles (spatial compounding) [2], applying pulses with different temporal frequencies (frequency compounding) [3], and, which has fairly recently been suggested, applying Thomson's multitaper approach [4], originally devised for power spectrum estimation. These methods reduce the speckle during the acquisition phase. They are based on the physical principle of averaging images with uncorrelated or partly uncorrelated speckle patterns. To acquire such uncorrelated images, one either has to increase the acquisition time by using several independent transmits (decrease frame rate), increase the sensor complexity by adding more receive apertures, or divide the existing system resources (aperture or frequency band), causing a reduction in the spatial resolution of the system. This again causes image-structural changes like cyst filling, swelling of hyperechoic structures and smearing of point-like targets [5], [6]. Recently, deep learning has been proposed as an approach to remedy this [7]. The second category of speckle reduction techniques contains a wealth of adaptive and non-adaptive image filtering approaches applied after the acquisition and initial beamforming, see e.g. [5], [8], [9] and references therein. Compared to the compounding approaches, the post image-formation filtering approaches may give stronger speckle suppression without compromising the spatial resolution [6], although with a risk of over-smoothing and potentially changing the true image anatomy [5]. Applying a speckle reduction technique from one of these categories does not exclude applying techniques from the other. Actually, combining techniques from the two categories seems to provide superior results and a possibility of controlling the limitations of the different approaches [6], [10]. Speckle reduction is an active field of research with several hundred papers published each year. These papers offers to a great degree refinements and extensions of compounding and post image-formation filtering approaches, defining as whole the state-of-the-art of speckle reduction.

The point spread function (PSF) of an acquisition system determines the system's target resolvability [1]. Several of the image-compounding approaches comprise splitting the lateral transducer aperture into several sub-parts, and letting each of them be the basis for one of the subsequently compounded images. Imaging with a smaller lateral aperture decreases the lateral resolution of the imaging system. The axial resolution, however, is determined by the bandwidth of the applied transmit pulse. In ultrasound imaging, the lateral resolution is often the most limited. Splitting the lateral aperture into parts, and using them independently for imaging, hence reduces the already limited spatial resolution, which necessarily degrades the final image.

In this paper we build upon the authors' previous work on non-adaptive, receive-side compounding [4] and study how some of these image degradations can be mitigated by combining the compounding with adaptive steering of the transducer-array at reception. We will limit our study to that of one-dimensional arrays, and to receive compounding, where, unless multiple receive transducers are employed (e.g. [11]), the different images comprising the compounds come from weighting differently the array elements upon reception. Receive compounding, as opposed to transmit and receive compounding, does not increase a system's acquisition time, and hence is useful also in high frame-rate settings. Moreover, 
we will not apply any temporal frequency compounding; the weights will be applied spatially as array tapers. In effect, this limits us to study changes laterally in the images. We show that the proposed approach of adaptively-steered compounding gives comparable speckle reduction to that of its non-steered counterpart, while it maintains, and even possibly improves, the visual sharpness of edges and point-like targets.

In the next section we begin by recapitulating the basics of ultrasound image formation and receive compounding for speckle reduction. Section III explains the proposed adaptively-steered receive compounding in detail. Section IV provides details of the conducted experiments, while Sections $\mathrm{V}$ and VI show and discuss the results, respectively. Concluding remarks can be found in Section VII.

\section{BACKGROUND}

\section{A. Image reconstruction}

A typical 2D phased array ultrasound imaging system forms images by sequentially transmitting a series of focused pulses into the medium of interest. The echoes of each pulse are recorded using an array of transducers. We will assume that we have a uniformly spaced linear array (ULA) for both transmitting the pulses and receiving the returning signals. For each image sample covered by the pulse, the recorded trace from each array element is time delayed to allow coherent summation of possible echoes stemming from that particular location.

Let the $M$-element column vector x contain such a timedelayed (focused) and range-gain compensated $M$-element array output for a location $(x, y)$ in the image plane. We assume that $\mathrm{x}$ stem from $\mathrm{I} / \mathrm{Q}$ modulated data and is hence complex-valued. The corresponding image-amplitude sample is:

$$
I_{x, y}=\left|\mathbf{w}^{T} \mathbf{x}\right|,
$$

where $\mathbf{w}$ is a weight vector that will either have uniform elements or be tapered by e.g. a Hamming window. The tapering permits a trade-off between spatial resolution, noise attenuation and sidelobe suppression. In the experiments, we will refer to images created using (1) with uniform weights as that of a delay-and-sum (DAS) beamformer.

\section{B. Receive compounding}

One can reduce the speckle level by averaging images with different speckle realizations. In receive compounding, as opposed to transmit-receive compounding, these images are formed by merely applying different array weights, or tapers, in (1). The resulting images are then incoherently averaged, or compounded, to form the final image. If we have $N_{t}$ such tapers, $\mathbf{w}_{i}, 1 \leq i \leq N_{t}$, we get the following for a given image-amplitude sample:

$$
I_{x, y}^{R C}=\sqrt{\sum_{i=1}^{N_{t}}\left|\mathbf{w}_{i}^{T} \mathbf{x}\right|^{2}} .
$$

We will be studying three approaches to forming these tapers, referred to, as in [4], as the Spatial, Welch and Thomson approach: a) Spatial: This approach synthesizes a set of shorter, non-overlapping sub-apertures. Each image in the compound is formed by using a separate sub-array at reception. An example having $N_{t}=3$ can be found in Figure 1a).

b) Welch: This is closely linked to the Spatial approach, as we again form shorter sub-apertures. Here, however, we allow weighted and overlapping tapers. The name is a reference to Welch's method in spectrum estimation [12], [13]. The particular version that we will be using consists of Hamming windows with $50 \%$ overlap. An $N_{t}=3$ example can be found in Figure 1b).

c) Thomson: Here the tapers are the ones that have the highest spectral concentration. In our setting, this means that we use the tapers that, combined, extract as much as possible the energy impinging on our array from within a given angular interval (cf. mainlobe) relative to that of the entire angular interval. The tapers are known as discrete prolate spheroidal sequences (DPSS), or Slepian sequences, and can be found by calculating the eigenvectors of the $M \times M$ matrix

$$
Q_{i j}=\frac{\sin \left[k_{c}(i-j)\right]}{\pi(i-j)}, \quad 1 \leq i, j \leq M,
$$

where $M$ again is the number of array elements and $k_{c}$ is a parameter guiding the width of the central angular interval [14]. The eigenvalues give the spectral concentration of the corresponding eigenvectors. Depending on the $k_{c}$ parameter (width of the mainlobe), a certain number of the eigenvalues will be close to one, having high spectral concentration, while, if sorted ascendingly, the following eigenvalues will quickly approach zero. The eigenvectors corresponding to the close-to unit eigenvalues are the ones used as tapers. When we assume the propagation of narrowband plane-waves and a one-half wavelength array element spacing, the $k_{c}$ parameter is given in wavenumbers, or the component of the wavenumber that corresponds to the spatial extent of the array. An example of a $N_{t}=3$ set of tapers can be found in Figure 1c).

The narrowband, plane-wave beampatterns corresponding to the sets of tapers in Figure 1 can be found in Figure 2. Note that all of the approaches have much wider mainlobes, hence yielding lower spatial resolutions, compared to that of the DAS approach shown in Figure 3. We refer the reader to [4] for more details and a review of the different approaches.

\section{ADAPTIVE RECEIVE-SIDE COMPOUNDING}

The idea behind adaptive receive-side compounding (ARC), is to adaptively - and for each image-sample independently steer the sensitivity of the array in the azimuthal direction by phase shifts as part of the compounding. In effect, we let the beampatterns of the array shift slightly in order to adapt it to the impinging signal. In this way it should be possible to avoid, or at least mitigate, the smearing of edges and point targets that is caused by the elongated widths of the effective mainlobes seen in receive-compounding settings.

Stated more precisely, let $\mathbf{a}_{\theta}$ be a steering vector that steers the array beampattern by an angle $\theta$. That is, if we assume a ULA with a one-half wavelength pitch, we have

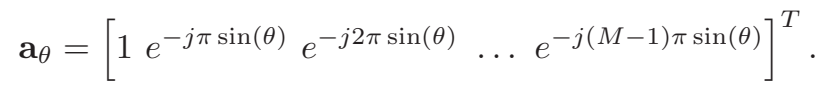




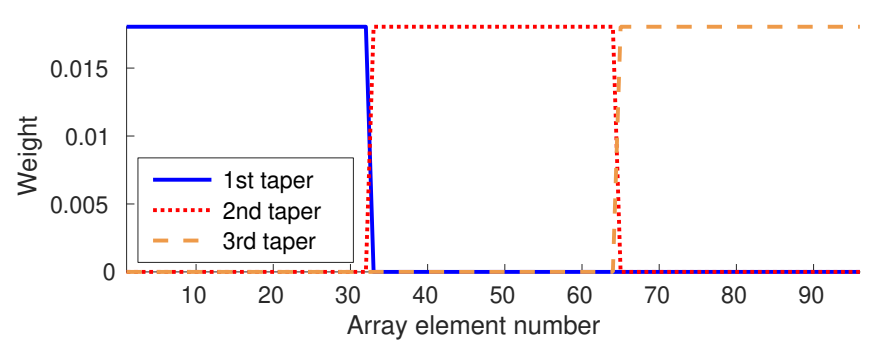

(a)

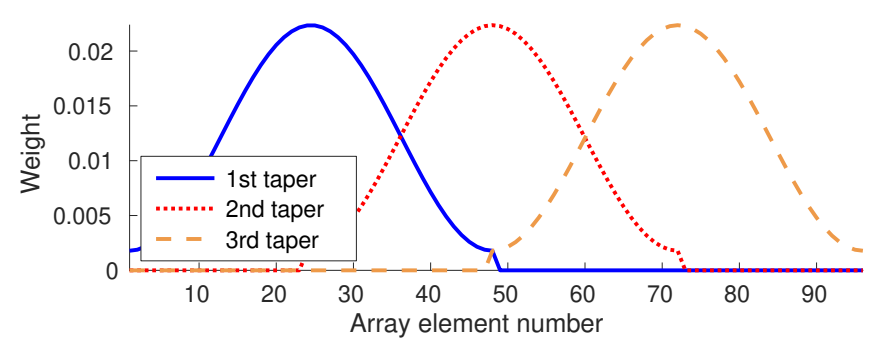

(b)

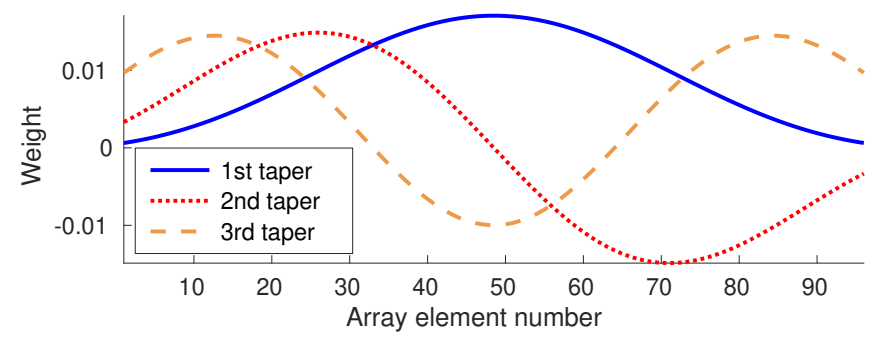

(c)

Figure 1. Example $N_{t}=3$ taper sets in the case of a 96-element ULA: a) Spatial, b) Welch and c) Thomson approach.

The steered image-amplitude sample output, cf. (2), then becomes:

$$
I_{x, y}^{R C}(\theta)=g_{\theta} \sqrt{\left.\sum_{i=1}^{N_{t}} \mid\left(\mathbf{w}_{i} \circ \mathbf{a}_{\theta}\right)^{T} \mathbf{x}\right)\left.\right|^{2}},
$$

where $\circ$ denotes the Hadamard (pointwise) product, and where we have added a steering-dependent gain factor, $g_{\theta}$. The gain factor is necessary to implement a "distortionless response" criterion similar to that of the Capon beamformer [15], i.e., a uniform at-zero-degree response (or, a uniform response for a single scatterer at the physical focus point) across $\theta$ values. The idea of adaptively steering the receive beams and applying a distortionless response criterion has previously been applied successfully in acoustical beamforming to various imaging setups [16]-[18]. It has proven as a robust and efficient approach to obtain Capon-like performance.

Since each of the slightly-steered, receive-compounding outputs has an identical at-focus response, and we assume that additional ("noise") scatterers contribute positively, we choose the final image-sample to be the minimum of these steered responses:

$$
I_{x, y}^{\mathrm{ARC}}=\min _{\theta \in[-\delta, \delta]} I_{x, y}^{R C}(\theta)
$$

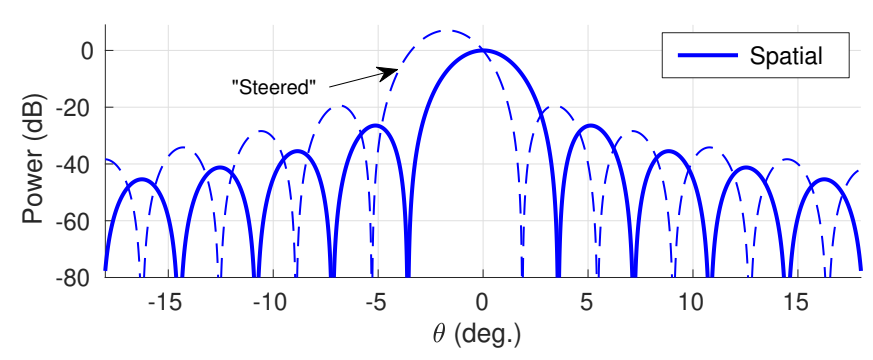

(a)

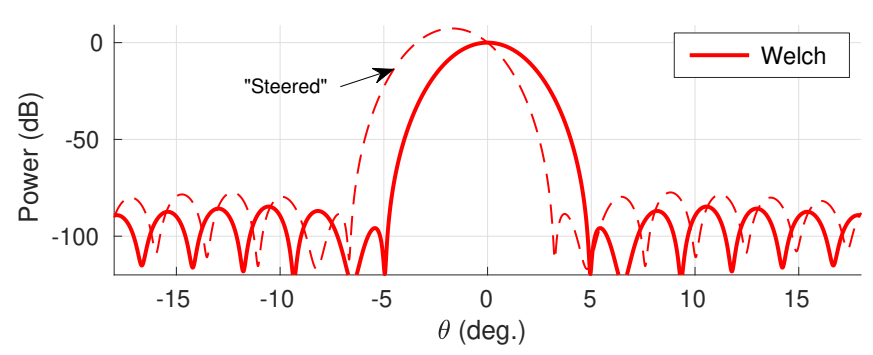

(b)

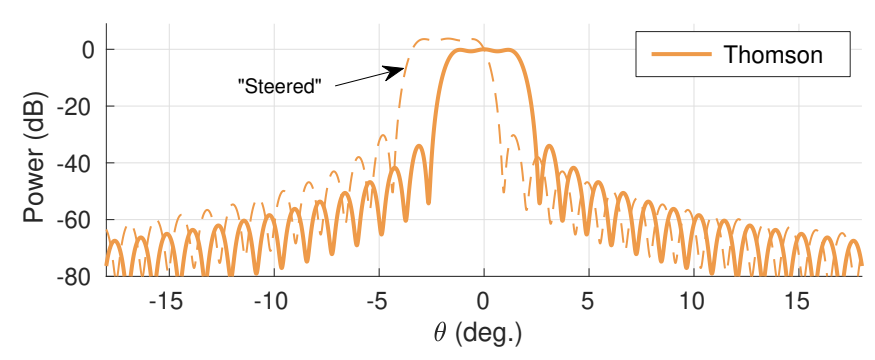

(c)

Figure 2. The corresponding beampatterns of the taper sets in Figure 1 when assuming a one-half wavelength pitch. The dashed lines show examples of responses that are angularly steered and where the gain has been increased to ensure a unit at-zero-degree response.

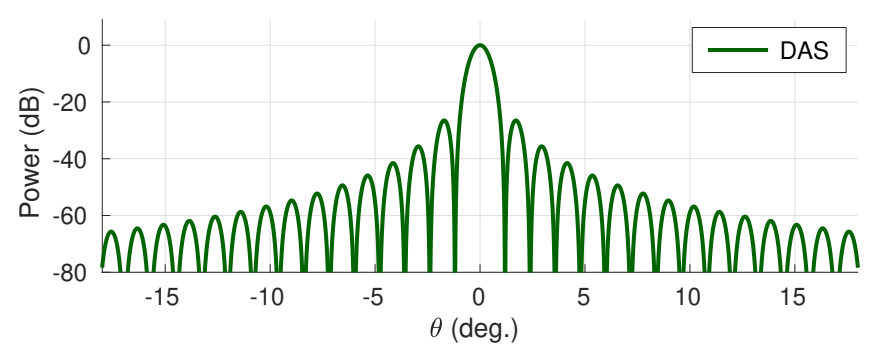

Figure 3. The beampattern of a uniformly weighted 96-element array when assuming a one-half wavelength pitch. Note the narrower mainlobe compared to those of the compounding approaches shown in Figure 2.

In (5) we have added a maximum allowed angular steering, $\delta$, to allow studying how gradually increasing the amount of steering affects the resulting images.

An illustration of such micro-steered beampatterns for the discussed compounding approaches can be found in Figure 2. In these examples, where we have steered to the left (negative angles), a strong scatterer located slightly to the right (positive angles) has reduced influence. By adaptively choosing the steering angle as the focus (spatial location corresponding to the zero frequency) moves, the effective "smearing" of such a 
scatterer is hence reduced. Notice how the gain factor ensures a unit at-zero-degree response also when steering is applied.

It might, dependent on the imaging setup, be necessary for the gain factor to have an additional component. Speckle can, in expectance, be seen as something impinging upon the array having a uniform power across angles. When applying steering in settings where we have background speckle, we would want the output power of the background (in expectance) to be independent of the steering angle. This would not automatically follow in scenarios where we transmit rather narrow beams, and where the effective beampattern could be steered partially outside the main intensity area of the transmitted pulse. In such cases it makes sense to first compensate for this effect by ensuring that we have a uniform speckle response over $\theta$ in expectance. When this is in place, we can add the gain ensuring a uniform at-zero-degree single-point response. What we end up with is an overall gain,

$$
g_{\theta}=g_{1 \theta} g_{2 \theta},
$$

where e.g. $\left.g_{1 \theta}=\left.\left(\sum_{i=1}^{N_{t}} \mid\left(\mathbf{w}_{i} \circ \mathbf{a}_{\theta}\right)^{T} \mathbf{1}\right)\right|^{2}\right)^{-\frac{1}{2}}$ ensures a fixed at-focus single-point response, while $g_{2 \theta}$ compensates for loss caused by steering partially outside the main intensity area of the transmitted pulse. For a given imaging setup this latter gain factor can be calculated based on transmit-receive beampattern overlaps. In principle, then, it will be range dependent (beyond that of the range-gain compensation already applied to the data), although in some imaging settings such a range effect would be small. One such setting would be the one we limit ourselves to; ULAs in combination with using the full array for all ranges. A practical estimate of $g_{2 \theta}$ can be obtained by fixing $g_{1 \theta}$ and ensuring uniform output power in speckle areas. This is the approach studied in this paper.

\section{EXPERIMENTS}

To study the effects of ARC we have run experiments on simulated speckle data using Field II [19], [20] as well as data stemming from phantom and in vivo acquisitions. The simulated data are especially suited to study spatial image structure distortions since we do not need to rely on a single speckle realization, which might invalidate the results. From the phantom data we verify both visually and by calculating image quality metrics that steering the compounds do improve the rendering of point-targets and cyst-like structures while not significantly deteriorating the amount of speckle reduction. Because of a lack of a "ground truth" and the limited scope of the study, the in vivo example images are solely visually studied and assessed by non-clinical experts.

In high frame-rate systems, a set of fewer, although broader, transmit lines in combination with multiple receive lines are often used to gain frame rate [21]-[23], as the framerate is ultimately limited by the number of transmitted pulses used to form each image. In the experiments, except for on the in vivo data, we have avoided using multiple line acquisition and instead relied on transmitting a rather high number of pulses to provide the necessary spatial image sample rate. This was done to avoid cluttering the results by discussing specific implementational issues and possible artifacts caused by upsampling at reception. In the in vivo experiment, however, keeping with a limited number of transmit pulses is unavoidable. Broad transmit beams were created by transmitting only using the central half of the available array.

\section{A. Tapers and compounding details}

We have limited our experiments to the study of three-way compounding $\left(N_{t}=3\right)$. The results in [4] indicate that having more tapers provide limited additional speckle reduction for the given transducer geometry. The tapers depicted in Figure 1 are the ones applied in the experiments.

The tapers in the Thomson approach are formed by extracting the principal $N_{t}=3$ eigenvectors of (3) for $k_{c}=$ $\pi\left(N_{t}+1 / 2\right) / M$, where $M$ is the number of array elements. Each taper is scaled by the corresponding eigenvalue. This provides the same tapers and method of application as in [4].

The steering-dependent gain, the $g_{\theta}$ in (4), was given an extra factor, $g_{2 \theta}$, as explained in Section III, to compensate for the use of directed transmit beams. Here, $g_{2 \theta}$ was set by fixing $g_{1 \theta}$ and ensuring a uniform full-image mean output power over $\theta$. We applied a brute-force, finely-gridded search with a sample distance just below 0.2 degrees when solving (5) for each image sample.

\section{B. Simulated speckle data}

The imaging setup for the Field II simulations consisted of a 96 -element array with a one-half wavelength pitch, a center frequency of $3.5 \mathrm{MHz}$ and a relative bandwidth of $92 \%$. A single-period sine was used as excitation, and the transmit focus was set to $80 \mathrm{~mm}$. 485 transmit and receive beams were distributed within $\pm 30^{\circ}$.

The starting point for creating the speckle scenes was placing randomly a dense set of scatterers uniformly in front of the transducer with a range and angular coverage larger than the scene boundaries. Two scenarios were simulated: one in which anechoic structures were created by removing the scatterers within cylinders of diameters $10 \mathrm{~mm}$ and $20 \mathrm{~mm}$, and one in which the amplitude of the scatterers within these same regions was increased by $30 \mathrm{~dB}$. A total of 27 speckle realizations were simulated. Figure 4 shows an example of a DAS image from one of these realizations where the scatterers inside the cylinders are removed. The hyperechoic cylinder images look similar, except for the dark, circular regions being replaced by hyperechoic $30 \mathrm{~dB}$ structures. In the images having anechoic structures, a single bright-point was added on top of the speckle. The width of the anechoic structures was estimated by taking the average of several of these images, extracting azimuthal scanlines at a range of $80 \mathrm{~mm}$, and then thresholding the result at a $-12 \mathrm{~dB}$ below the background amplitude. The width of the hyperechoic cylinders were similarly estimated, although the threshold was then $12 \mathrm{~dB}$ below the $30 \mathrm{~dB}$ level of the cylinders. To get an idea of the variability of the estimates, this was done repeatedly with the images selected for averaging drawn randomly with replacement, cf. bootstrapping. For easier comparison of the different approaches, the resulting images were all scaled to have similar background intensity. 


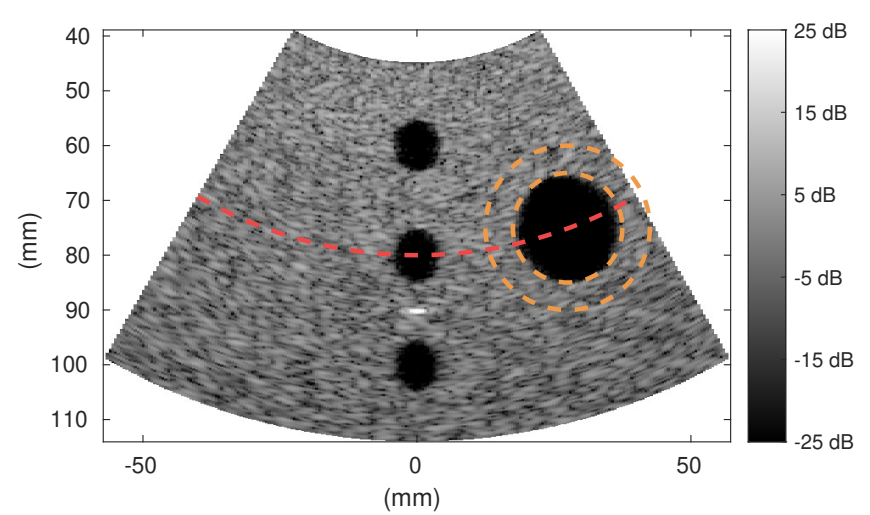

Figure 4. Example of the simulated cysts-in-speckle images rendered using DAS. The location of the $80 \mathrm{~mm}$ range image cuts used for estimating the widths of the cylinders is superimposed as a stippled line. The anechoic region and its background used in calculating gCNR are also indicated.

\section{Recorded phantom data}

Individual channel-data were acquired using a 96-element 1.5D phased array transducer attached to a specially adapted GE Vivid E9 scanner (GE Healthcare, Waukesha, WI). A Gammex 403GS LE multi-purpose phantom (Gammex RMI, Middleton, WI) was used as the imaging target. The center frequency of the transmitted pulse was $3.5 \mathrm{MHz}$ and the beams had a transmit focus at a range of $80 \mathrm{~mm}$. Both dynamic azimuth and elevation focusing was used on reception. A total of 432 transmit and receive beams were swept within $\pm 37.5^{\circ}$. The difference between a $1.5 \mathrm{D}$ and a $1 \mathrm{D}$ probe is in their ability to do elevation focusing. In our experiments where we study lateral resolution, this should in general not impact the results.

\section{In vivo data}

Channel data from a parasternal long-axis view of the heart of a healthy volunteer were acquired using the Verasonics Vantage 256 system (Verasonics, Kirkland, WA, USA). The probe used was the Verasonics P4-2v 64-element, 0.3-mm pitch, phased array probe. The images were acquired in fundamental mode. The transmit center frequency was $3 \mathrm{MHz}$ and the data were sampled at four times the center frequency. The transmit focus was placed at $52 \mathrm{~mm}$ and each image was created from 101 focused transmit beams, covering a sector scan from $-37.5^{\circ}$ to $37.5^{\circ}$. The images were acquired by a trained professional and written consent from the volunteer had been acquired ${ }^{1}$. The channel data were stored and then processed using the UltraSound ToolBox [24].

\section{E. Image quality assessment}

When studying speckle reduction in tissue images, two common figures of merit are 1) the speckle signal-to-noise

\footnotetext{
${ }^{1}$ In connection with the data recording we received a Letter of Exemption from the Regional Committee for Medical \& Health Research Ethics in Norway with Institutional Review Board Reference No. IRB00001870 REK because they "found the Research Project to be outside the remit of the Act on Medical and Health Research (2008)."
}

ratio and 2) the contrast-to-noise ratio. The speckle signalto-noise ratio is defined as the ratio between the produced image amplitude mean value $\mu$ and its standard deviation $\sigma$ in homogeneous regions:

$$
\mathrm{SNR}=\mu / \sigma .
$$

In fully developed speckle, where the pixel value follows a Rayleigh distribution, the SNR can theoretically reach $1.91 \sqrt{N}$, where $N$ is the number of uncorrelated images that are combined.

The applied metric for contrast-to-noise is that of the recently-proposed generalized contrast-to-noise ratio ( $\mathrm{gCNR}$ ) [25]. The gCNR is chosen because it has been proven robust for signal statistic alterations imposed by various adaptive beamforming algorithms. gCNR is a measure of lesion detectability. It measures the success rate of an optimal detector at the task of separating pixel areas with different backscatter coefficients, often referred to as healthy and pathological pixels within lesion detectability. It is calculated as

$$
\mathrm{gCNR}=1-\mathrm{OVL},
$$

where OVL is the relative overlap area between the normalized pixel histograms of the anechoic/hyperechoic and background regions.

\section{REsults}

\section{A. Simulated speckle data}

Figures 5 and 6 show example images generated from the simulated cyst data. We see that the amount of speckle has been reduced in all of them compared to that of the DAS image in Figure 4. When applying adaptive steering, both the anechoic and hyperechoic structures become more round and more true to that of the actual model. The greatest effect of allowing adaptive steering is clearly for the Thomson approach. Here the circular structures become markedly more round and the structure borders appear more steep and welldefined. When visually studying the speckle patterns, however, that of the steered Thomson appear to be a bit more finegrained.

Figure 7 shows quantifiably how the different approaches change the estimated azimuthal widths of the circular structures; both the anechoic and their hyperechoic twins. As the amount of allowed steering is increased, all the compounding approaches improve on their estimates; anechoic structures become wider and more correct, and likewise, although in an opposite manner, the regions of hyperechoic also become more correct. The steered Thomson compounding provides even better estimates than that of the non-compounded DAS approach.

Figure 8 shows azimuthal image cuts. We clearly see that by allowing steering the single bright-point becomes much more point-like, especially for the Thomson approach. The image cuts through the anechoic and hyperechoic structures also demonstrates that by allowing steering the swelling of the hyperechoic structures and the filling of their anechoic twins are reduced. 
Figure 9 shows SNR and gCNR, as defined in Section IV-E. The SNR was calculated from a speckle region located to the left in the images, while the specific anechoic and background regions used in the gCNR calculations are superimposed in Figure 4b). The gCNR curves show a clear increase when applying steering, indicating that they produce images where detecting cysts becomes easier. On the other hand, allowing steering do decrease the SNR; however, the speckle reduction capabilities of the compounding approaches are still high, as the SNR values of the steered approaches are much higher than that of the DAS images.

The necessary allowed steering needed to make the compounding approaches reach their full potential differs between the different compounding approaches, however further increasing the maximum allowed steering does not have a detrimental effect.

Figure 11 shows amplitude histograms from speckle regions. We see that the amplitude distributions from all the compounding approaches are very similar, although the steered versions are indeed slightly less peaked compared to their nonsteered counterparts.

More as a supplementary note, the gain functions $g_{\theta}$ in (4) can be found in Figure 10. We see, as expected, that the gain function used by the Thomson approach is more flat before it quickly peaks, while the other approaches has a more gradual increase.

\section{B. Recorded phantom data}

Figure 12 shows SNR and gCNR calculated from the resulting images after processing the recorded phantom data. The SNR was calculated from a speckle region centrally located at a range of about $80 \mathrm{~mm}$. The specific anechoic and background regions used in the gCNR calculations are indicated in Figure 13b). Qualitatively, the plots are very similar to the SNR and gCNR plots calculated from the simulated data; steering increases the gCNR while lowers the SNR. The SNR values do, though, still show a significant speckle reduction capability when steering is applied.

From the images in Figure 13 we see that by allowing steering the point-targets become much more point-like, especially for the Thomson approach. The cyst-like structure is, as in the simulations, more round and prominent when applying adaptive steering. Figure 14 shows some azimuthal image cuts at ranges $60 \mathrm{~mm}$ and $83 \mathrm{~mm}$. We see that the adaptively steered versions limit the growth of the edges into the anechoic regions, and also that they reproduce the pointtarget as a sharper, or more narrow, object. Again, this applies especially for the Thomson approach.

As for the simulated data, the different compounding approaches require a different amount of steering to achieve the full effect of steering, however, and also as seen for the simulated data, a further increase of the allowed steering does not have a detrimental effect.

\section{In vivo data}

Figure 15 shows images of a parasternal long-axis view made with DAS and un-steered and steered receive-side
Thomson compounding. The speckle in the two receive-side compounded images, (b) and (c), appear more smooth than the DAS image in (a). Comparing the two compounded images, point-like targets have larger lateral extend in the un-steered than the steered image. This can be observed in for instance the interventricular septum (the high-intensity area at ranges between $45 \mathrm{~mm}$ and $60 \mathrm{~mm}$ ) or at the defined scatterers at $($ range, lateral $)=(83 \mathrm{~mm}, 0 \mathrm{~mm})$ and $(95 \mathrm{~mm},-10 \mathrm{~mm})$. As in the simulated and phantom images, lateral edges do also appear more defined when steering is applied. Example of this can be observed in the edge in the center of the image at ranges $100 \mathrm{~mm}$ to $115 \mathrm{~mm}$.

The characteristics of the gain functions $g_{\theta}$ in (4) for these experimental data (not shown) are very similar to that of the phantom and simulated data, although, of course the abscissa shows larger angles because of the shorter aperture used.

\section{DISCUSSION}

From the results it is clear that applying adaptive steering when doing receive-side compounding does provide the predicted benefits: point-targets are reproduced as more narrow objects and the edges of cysts and hyperechoic regions are more correctly placed. The most prominent improvements, however, with estimates even better than that of the noncompounded DAS approach, can be found for the Thomson approach. This is related to its flat and angularly concentrated beampattern (cf. shape and width of the mainlobe), as can be seen in Figure 2. Such a beampattern with its sharp responsetransitions, needs only a limited amount of steering to moreor-less completely move the mainlobe away from interfering scatterers. Furthermore, such beampatterns can be steered with a limited amount of the scaling needed to ensure the at-zerodegree distortionless response, cf. Figure 10. This is important since such scaling would also scale the response of interfering or background scatterers, which in effect would limit the adaptability of the approach. It should be noted, though, that we have generally not improved the resolution as defined by the minimum distance between distinguishable objects. Steering at reception might make targets more sharp and trim off edges; however, creating dips between multiple targets where there were none before requires more than just steering. Steering might, nonetheless, be able to improve the apparent resolution in cases where the targets are already separable.

The first-order speckle statistics of the Thomson approach, cf. the pixel-amplitude histograms, do not show much change when allowing steering. The spatial distribution, or the spatial speckle pattern, however, do become a bit more finely grained. This is because the approach is able to adapt itself, to some extent, to the spatial variations within the speckle. Since the amount of speckle reduction (cf. SNR) and the firstorder statistics do not change too much, this should not necessarily be a drawback of the approach. However, the amount of change in the spatial speckle pattern introduced by adaptive steering can be lowered by reducing the upper limit of the allowed steering, i.e., reduce the overall steering effect. Limiting the amount of allowed steering could in an applied setting be a user-guided parameter. 


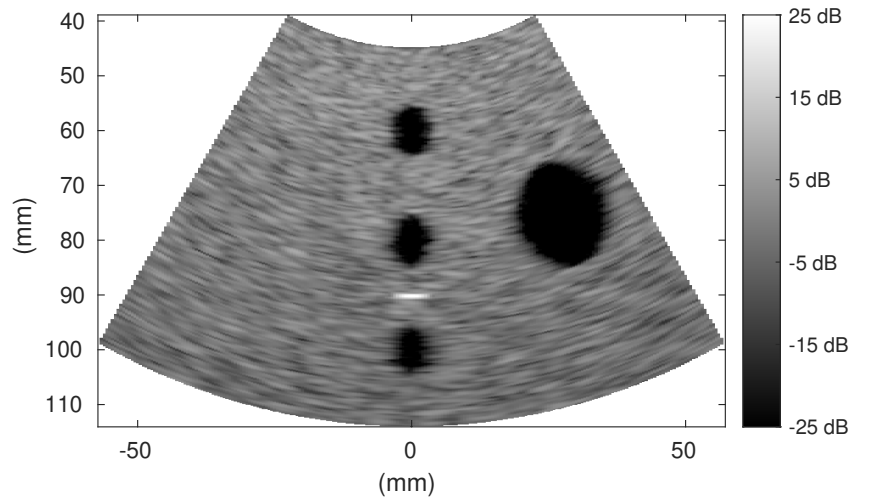

(a) Welch, no steering

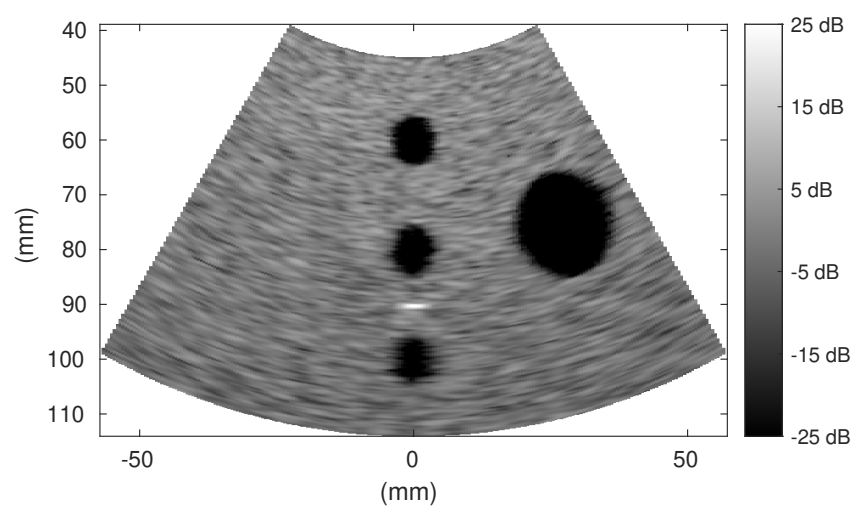

(c) Welch, adaptively-steered

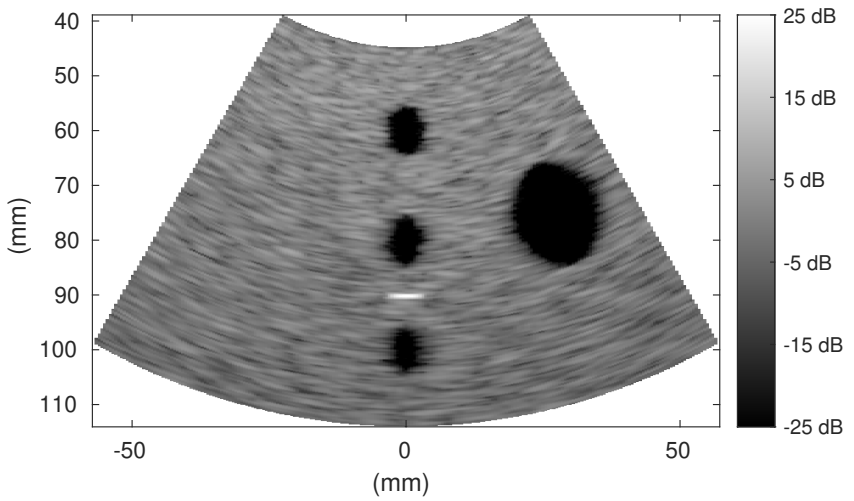

(b) Thomson, no steering

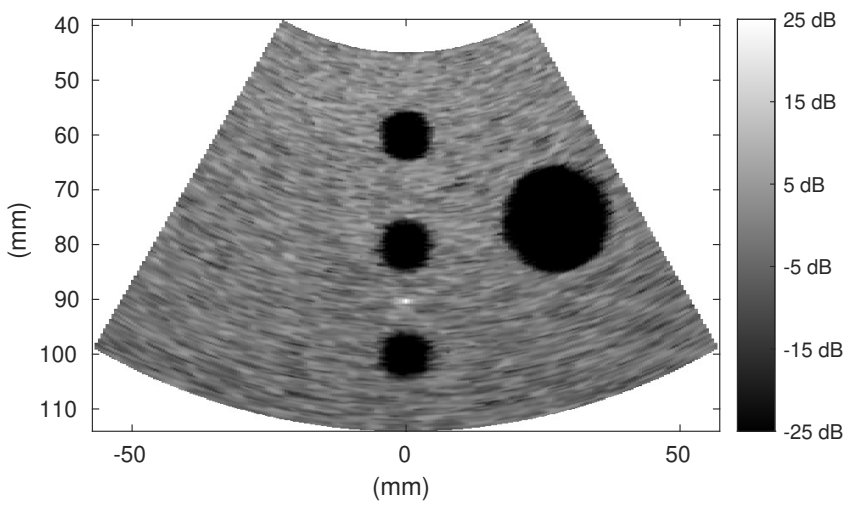

(d) Thomson, adaptively-steered

Figure 5. Example compound images from the simulated data where we have removed scatterers creating several circularly-shaped anechoic structures as well as added a single bright point.

The increase we see in cyst-detectability, cf. the increase in $\mathrm{gCNR}$, when applying adaptive steering comes from the more correct rendering of the circular cysts. Not blurring the depictions of cysts is of course important for their detection, however, one should note that if we had excluded the edges from the gCNR calculations, the contrast would not increase, merely stay the same as we allowed for adaptive steering.

The search over $\theta$ for the minimum of (5) has in the experiments been done using an overly fine grid. To reduce the amount of computations needed, other optimization techniques or a much coarser grid can be used. Even though this is outside the scope of this paper, preliminary results indicate that only a handful of steering angles are needed to get significant improvements. Even though the experiments show that including a larger angular search window in the adaptive steering does not cause detrimental effects on the resulting images, the interval should anyway, for computational benefits, of course be limited to covering the width of the beampattern mainlobe (cf. Figure 2).

A straight-forward extension of this work would be to study the effect of allowing not only steering, but qualitatively different tapers in the adaptive search process. As an example, one could choose among the outputs from steered tapers from both the Spatial, Welch and Thomson approach. This could e.g. possibly lead to reduced effects of sidelobes.

A further extension of this work would be to include adaptively steered temporal frequency compounding, similar in nature to the adaptivity presented herein. A combined approach would in essence introduce 2D PSF steering; much the same as most post-processing filtering approaches, and thereby render a more fair and direct comparison between these techniques. As of now, the proposed approach is most suited to be used as a modest speckle reduction approach with point and edge preservation capability, or as a tool applied in combination with a post-processing approach.

\section{CONCLUSION}

We have studied the effect of adaptively steering the angular sensitivity of the transducer array by spatial frequency modulation in combination with receive-side compounding for speckle reduction. The adaptivity is based on a minimumoutput distortionless response criterion, similar in nature to that of the Capon beamformer. The criterion makes sure that however we steer, the response for a scatterer at the spatial focus remains undistorted. The experiments - on simulated, phantom and in vivo data - show that the approach is capable of reducing some of the unwanted effects that typically come with such receive-side compounding, like the filling of cysts, swelling of hyperechoic regions and smearing of point-like image structures. All the studied compounding approaches responded positively to adaptive steering, although the Thomson multitaper approach, with its flat and highly concentrated beampattern, clearly benefited the most, although it yielded 


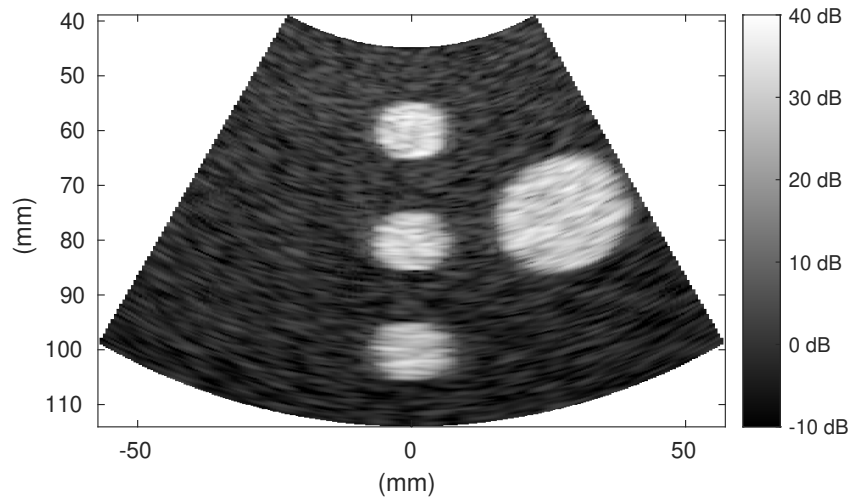

(a) Welch, no steering

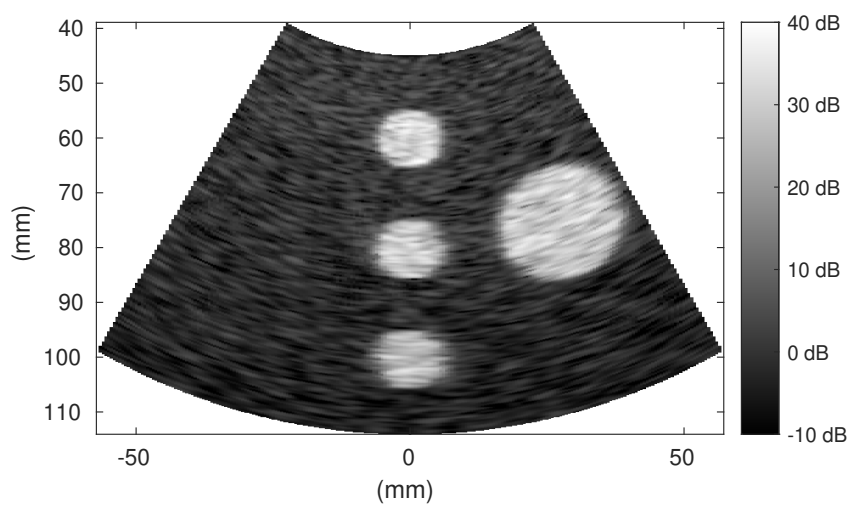

(c) Welch, adaptively-steered

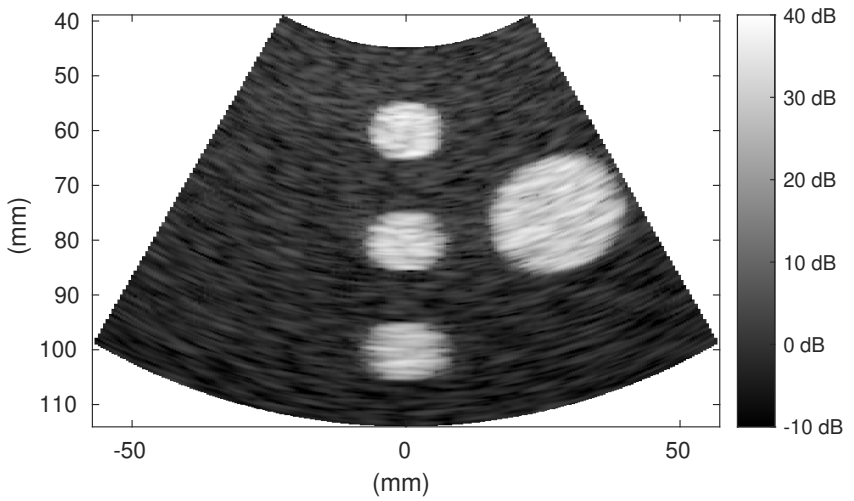

(b) Thomson, no steering

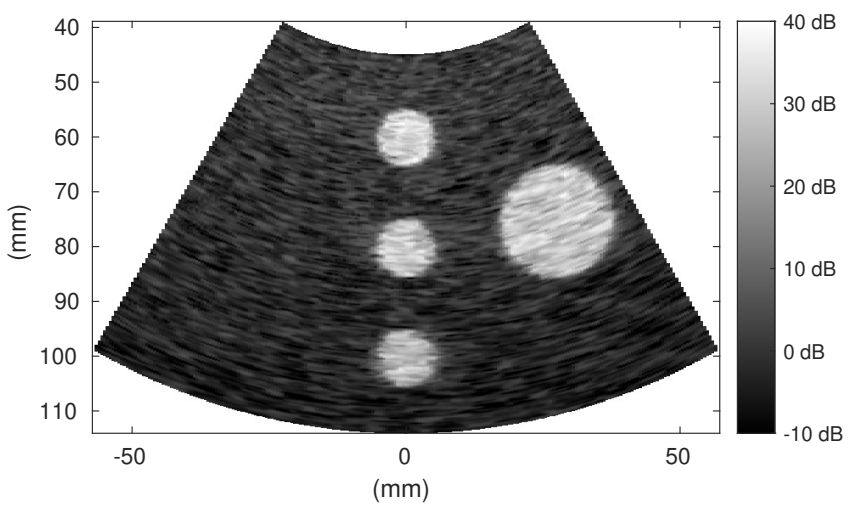

(d) Thomson, adaptively-steered

Figure 6. Example compound images from the simulated data where we have several circularly-shaped hyperechoic structures.

subtle changes in the spatial speckle patterns. Finding the optimal approach for minimizing the computational requirements is left as future work; however, even using a straight-forward, grid-based search strategy, the suggested approach comes at a moderate computational cost.

\section{ACKNOWLEDGEMENTS}

The authors would like to thank Prof. Fritz Albregtsen for much-appreciated feedback on an earlier draft of the manuscript, Dr. Anders Sørnes, GE Vingmed Ultrasound, for providing the experimental ultrasound phantom data, and Dr. Ole Marius Hoel Rindal for providing the in vivo data.

\section{REFERENCES}

[1] T. Szabo, Diagnostic Ultrasound Imaging: Inside Out, ser. Biomedical Engineering. Elsevier Science, 2013. [Online]. Available: https: //books.google.no/books?id=wTYTAAAAQBAJ

[2] C. B. Burckhardt, "Speckle in Ultrasound B-Mode Scans," IEEE Trans. Sonics Ultrason., vol. 25, no. 1, pp. 1-6, 1978.

[3] J. G. Abbott and F. L. Thurstone, "Acoustic Speckle: Theory and Experimental Analysis," Ultrasonic Imaging, vol. 1, no. 4, pp. 303-324, 1979.

[4] A. C. Jensen, S. P. Näsholm, C.-I. C. Nilsen, A. Austeng, and S. Holm, "Applying Thomson's multitaper approach to reduce speckle in medical ultrasound imaging," Ultrasonics, Ferroelectrics and Frequency Control, IEEE Transactions on, vol. 59, no. 10, 2012.

[5] S. H. Contreras Ortiz, T. Chiu, and M. D. Fox, "Ultrasound image enhancement: A review," Biomedical Signal Processing and Control, vol. 7, no. 5, pp. 419-428, 2012.
[6] D. Adam, S. Beilin-Nissan, Z. Friedman, and V. Behar, "The combined effect of spatial compounding and nonlinear filtering on the speckle reduction in ultrasound images," Ultrasonics, vol. 44, no. 2, pp. 166181, 2006.

[7] D. Hyun, L. L. Brickson, K. T. Looby, and J. J. Dahl, "Beamforming and speckle reduction using neural networks," IEEE Transactions on Ultrasonics, Ferroelectrics, and Frequency Control, vol. 66, no. 5, pp. 898-910, 2019.

[8] S. Finn, M. Glavin, and E. Jones, "Echocardiographic speckle reduction comparison," Ultrasonics, Ferroelectrics and Frequency Control, IEEE Transactions on, vol. 58, no. 1, pp. 82-101, 2011.

[9] R. Rosa and F. Monteiro, "Performance analysis of speckle ultrasound image filtering," Computer Methods in Biomechanics and Biomedical Engineering: Imaging \& Visualization, pp. 1-9, 2014. [Online]. Available: http://dx.doi.org/10.1080/21681163.2014.935803

[10] R. G. Barr, R. L. Maldonado, and D. Georgian-Smith, "Comparison of conventional, compounding, computer enhancement, and compounding with computer enhancement in ultrasound imaging of the breast," Ultrasound quarterly, vol. 25, no. 3, pp. 129-134, 2009.

[11] V. Behar, D. Adam, and Z. Friedman, "A new method of spatial compounding imaging," Ultrasonics, vol. 41, no. 5, pp. 377-384, 2003.

[12] P. D. Welch, "The use of fast fourier transform for the estimation of power spectra: a method based on time averaging over short, modified periodograms," IEEE Transactions on audio and electroacoustics, vol. 15, no. 2, pp. 70-73, 1967.

[13] J. G. Proakis, Digital signal processing: principles algorithms and applications. Pearson Education India, 2001.

[14] L. C. Barbosa, "A maximum-energy-concentration spectral window," IBM Journal of Research and Development, vol. 30, pp. 321-325, 1986.

[15] H. Van Trees, Optimum array processing. Wiley Online Library, 2002.

[16] J. Synnevåg, A. Austeng, and S. Holm, "A low-complexity datadependent beamformer," IEEE Transactions on Ultrasonics, Ferroelectrics, and Frequency Control, vol. 58, no. 2, pp. 281-289, 2011.

[17] A. E. Albright Blomberg, A. Austeng, R. E. Hansen, and S. A. V. Synnes, "Improving sonar performance in shallow water using adaptive 


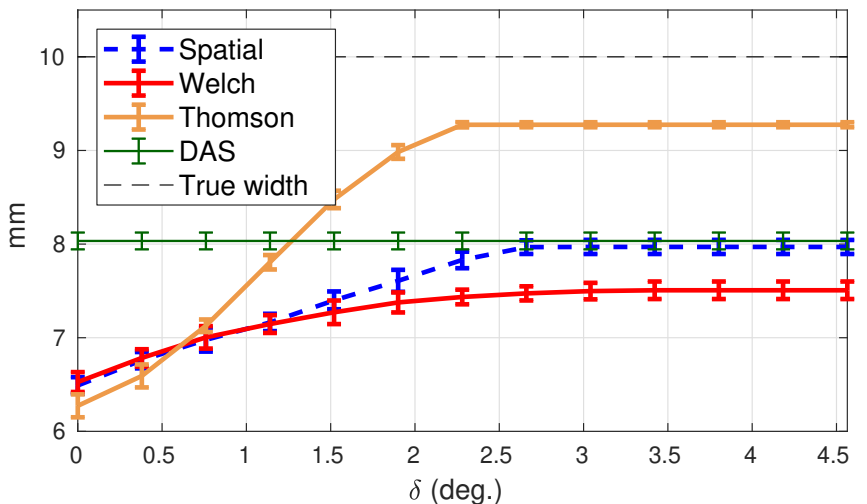

(a)

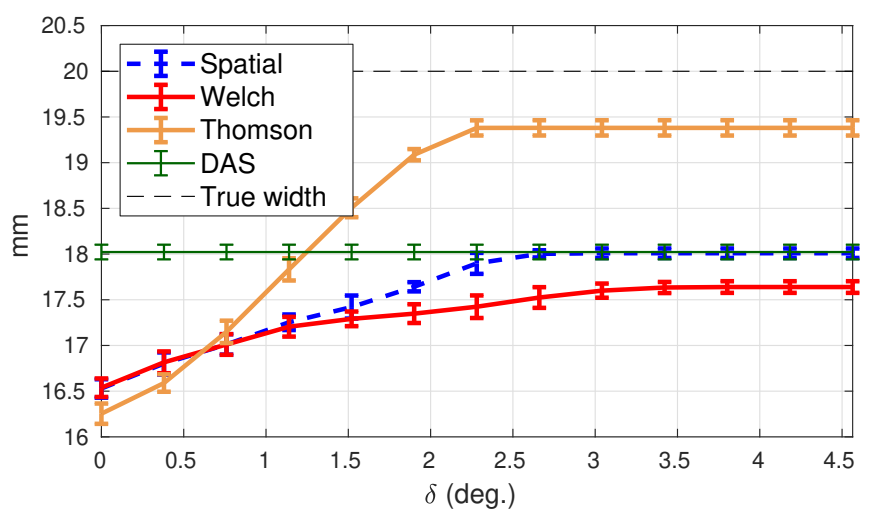

(c)

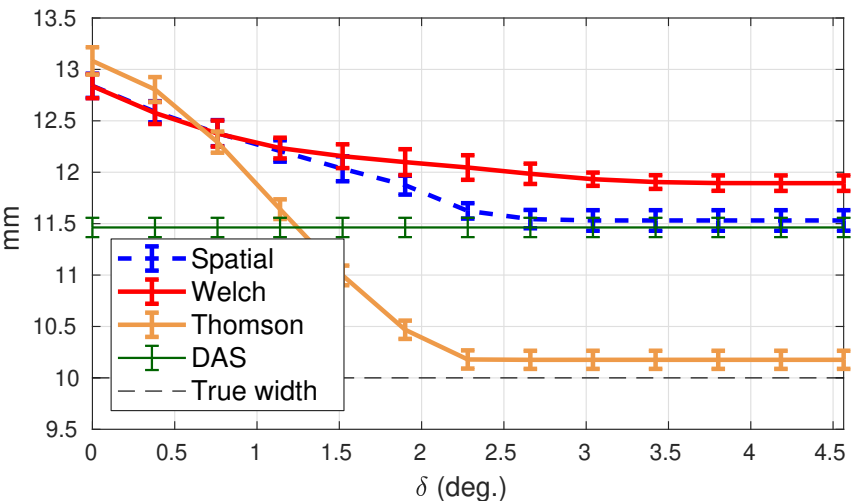

(b)

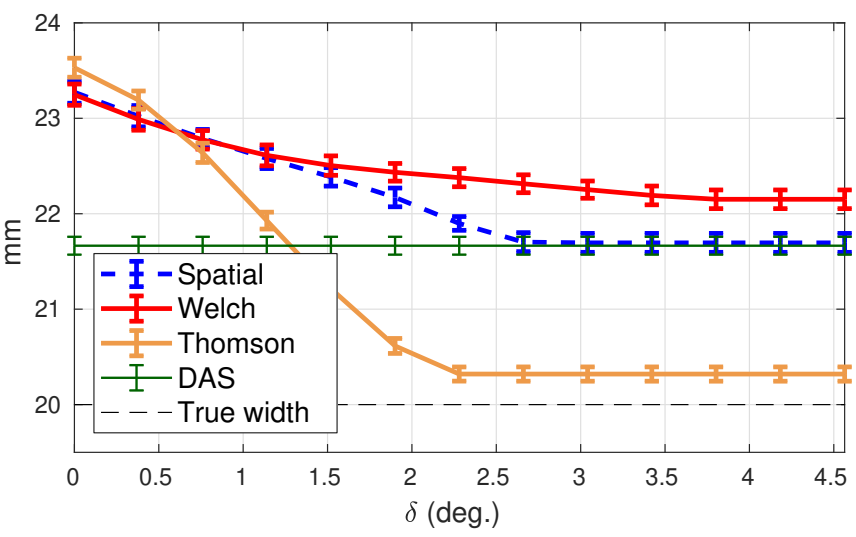

(d)

Figure 7. The estimated widths of the anechoic and hyperechoic structures in the simulated data, cf. Figure 4: a) and c) anechoic structures, while b) and d) hyperechoic structures. The vertical bars indicate one standard deviation. Along the abscissa we have the maximum allowed angular steering, cf. $\delta$ in (5).

beamforming," IEEE Journal of Oceanic Engineering, vol. 38, no. 2, pp. 297-307, 2013.

[18] J. I. Buskenes, R. E. Hansen, and A. Austeng, "Low-complexity adaptive sonar imaging," IEEE Journal of Oceanic Engineering, vol. 42, no. 1, pp. 87-96, 2017.

[19] J. A. Jensen, "Field: A program for simulating ultrasound systems," in 10th Nordic-Baltic Conference on Biomedical Imaging, vol. 4, supplement 1, PART 1: 351-353, 1996.

[20] J. A. Jensen and N. B. Svendsen, "Calculation of pressure fields from arbitrarily shaped, apodized, and excited ultrasound transducers," IEEE Trans. Ultrason. Ferroelectr, Freq. Control, vol. 39, no. 2, pp. 262-267, 1992.

[21] D. Shattuck, M. Weinshenker, S. Smith, and O. von Ramm, "Explososcan: A parallel processing technique for high speed ultrasound imaging with linear phased arrays," J. Acoust. Soc. Am., vol. 75, p. 1273, 1984.

[22] S. W. Smith, H. G. Pavy Jr., and O. T. von Ramm, "High-speed ultrasound volumetric imaging system-part I: Transducer design and beam steering," IEEE Trans. Ultrason. Ferroelectr, Freq. Control, vol. 38, pp. 100-108, 1991.

[23] O. T. von Ramm, S. W. Smith, and H. G. Pavy Jr., "High-speed ultrasound volumetric imaging system-part II: Parallel processing and image display," IEEE Trans. Ultrason. Ferroelectr., Freq. Control, vol. 38, pp. 109-115, 1991.

[24] A. Rodriguez-Molares, O. M. H. Rindal, O. Bernard, A. Nair, M. A. L. Bell, H. Liebgott, and L. Austeng, Andreas Løvstakken, "The ultrasound toolbox," in Ultrasonics Symposium (IUS), 2017 IEEE International. IEEE, 2017, pp. 1-4.

[25] A. Rodriguez-Molares, O. M. H. Rindal, J. D’hooge, S.-E. Måsøy, A. Austeng, M. A. L. Bell, and H. Torp, "The generalized contrastto-noise ratio: a formal definition for lesion detectability," IEEE Transactions on Ultrasonics, Ferroelectrics, and Frequency Control, 2019. 


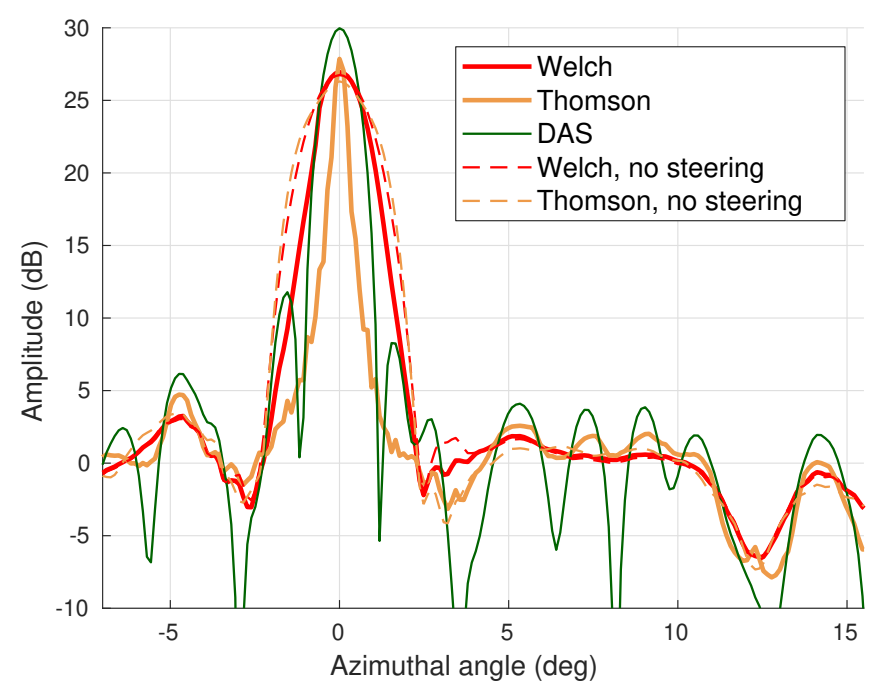

(a)

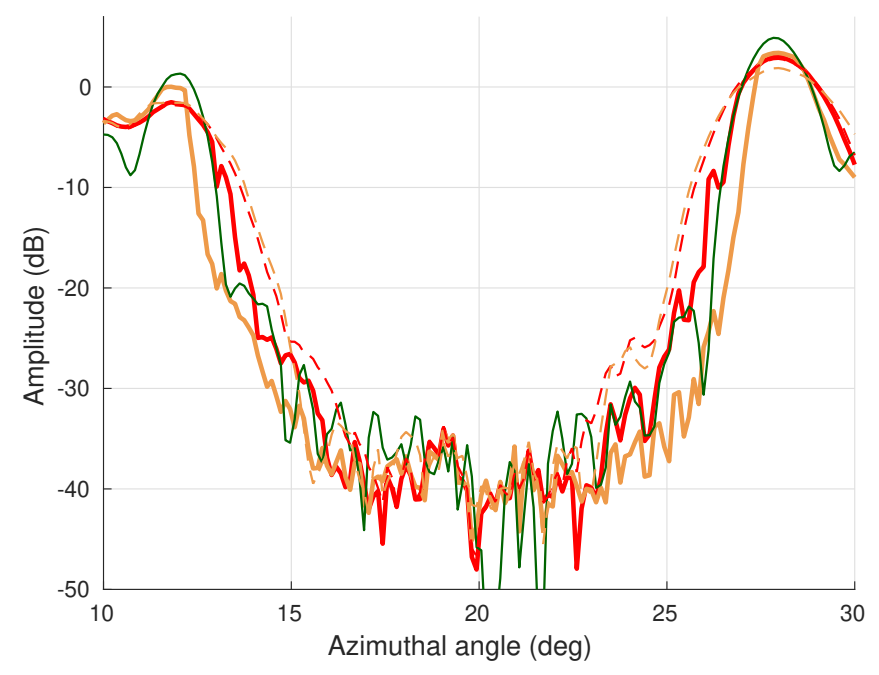

(b)

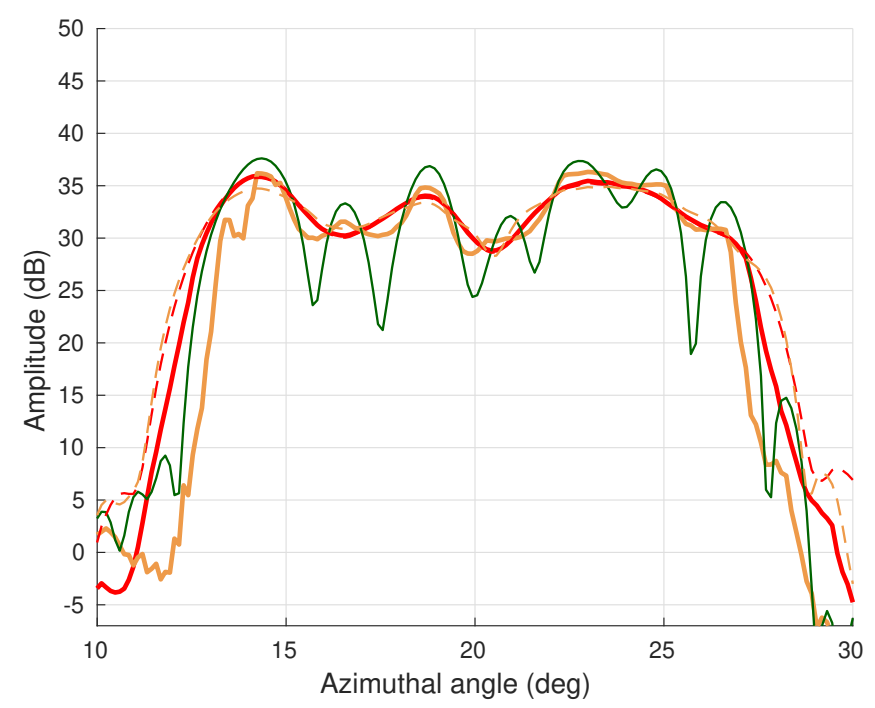

(c)

Figure 8. Azimuthal cuts from the simulated images seen in Figures 5 and 6. a) A cut at a range of $90 \mathrm{~mm}$ passing right through the single bright point. b) A cut at a range of $80 \mathrm{~mm}$ through a larger anechoic region. c) Same as b), although from images of hyperechoic structures.

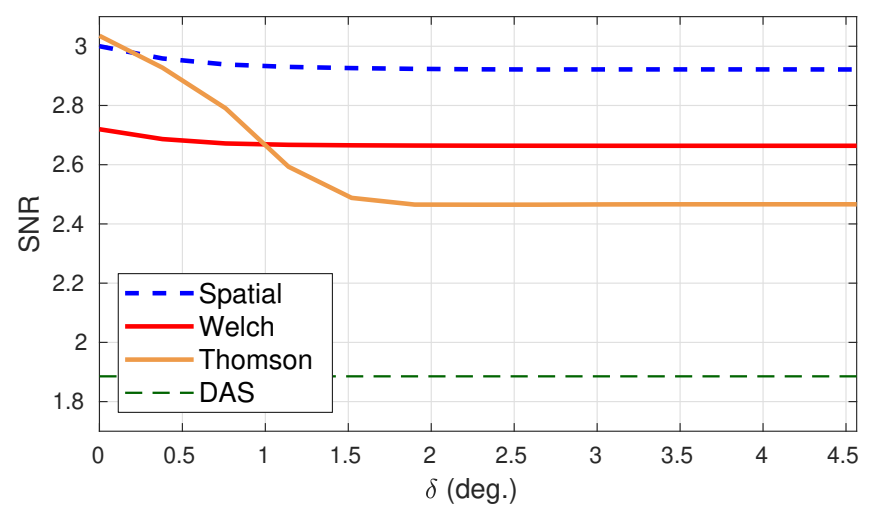

(a)

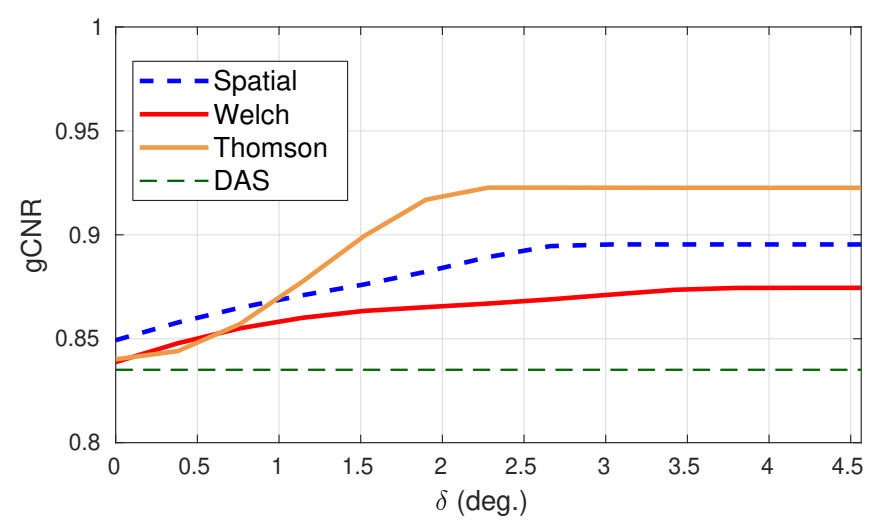

(b)

Figure 9. Image quality metrics calculated from the simulated data. Along the abscissa we have the maximum allowed angular steering, cf. $\delta$ in (5).

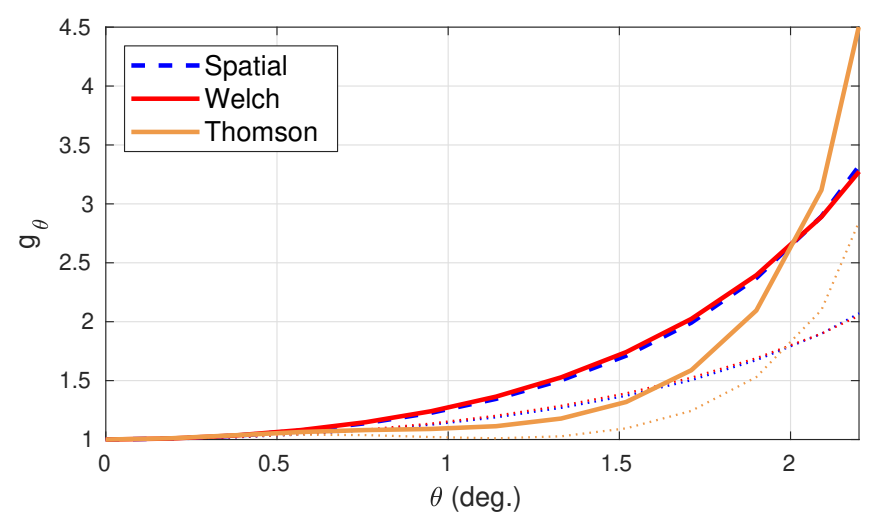

Figure 10. The gains ensuring distortionless responses as applied on the simulated data. The fine dashed lines are the part of the gain function that ensures a uniform at-zero-degree, plane-wave response, i.e., $g_{1 \theta}$ in (6). 


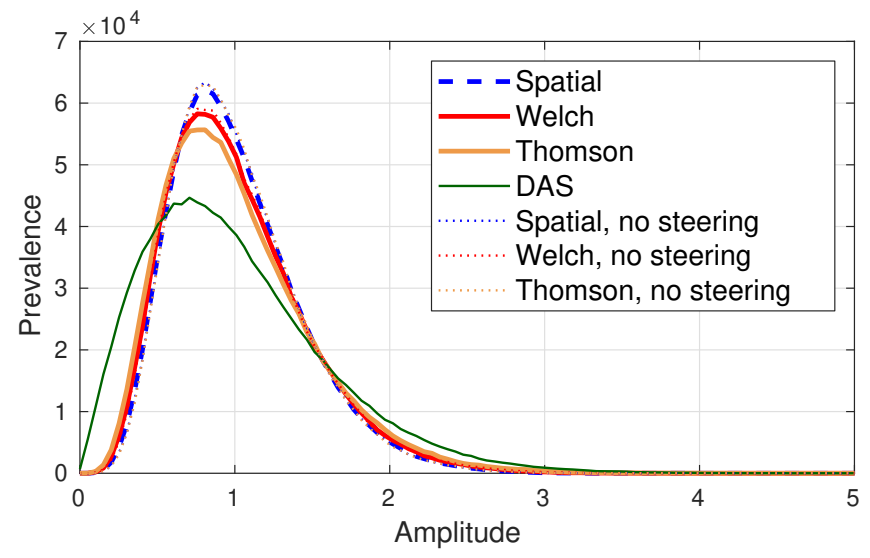

Figure 11. Pixel-amplitude histograms from speckle regions from the simulated data sets.

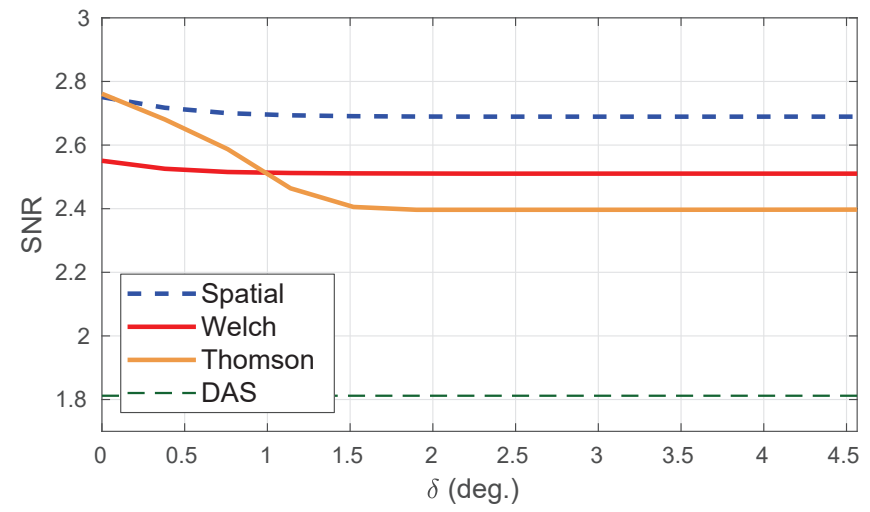

(a)

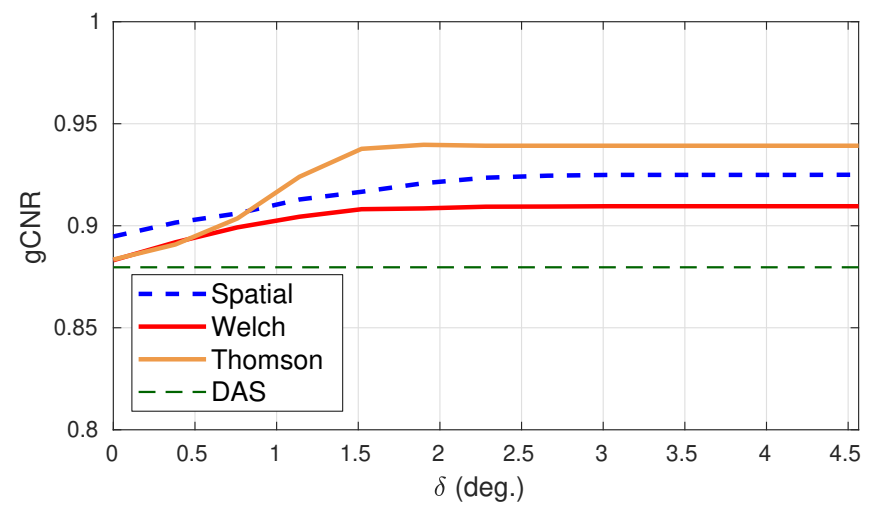

(b)

Figure 12. Image quality metrics calculated from the recorded phantom data. Along the abscissa we have the maximum allowed angular steering, cf. $\delta$ in (5).

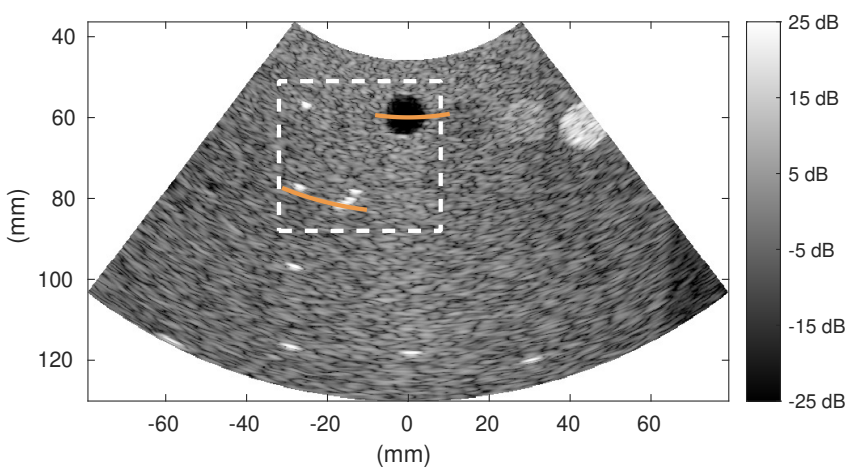

(a) DAS

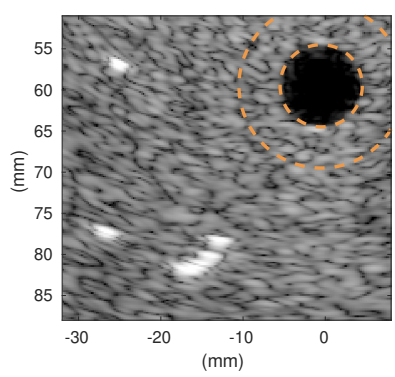

(b) DAS

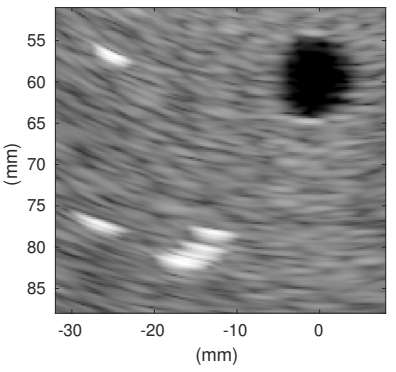

(c) Welch, no steering

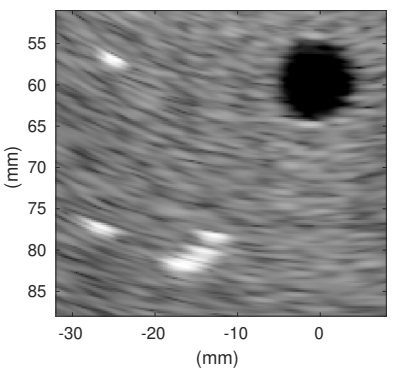

(e) Welch

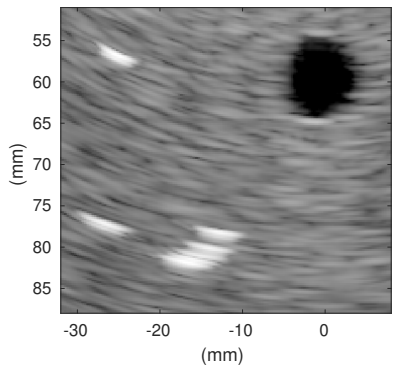

(d) Thomson, no steering

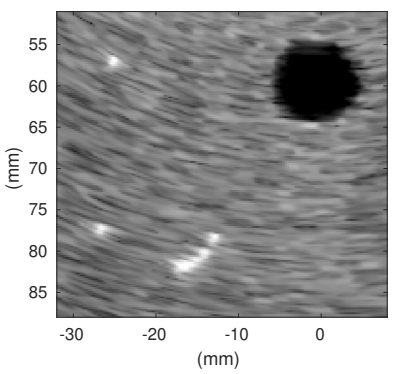

(f) Thomson
Figure 13. Recorded phantom data. a) A DAS-processed image with the image cuts of Figure 14 indicated. b) A magnified view of a) with the regions used for calculating gCNR indicated. c)-f) Receive-compounded versions of b). 

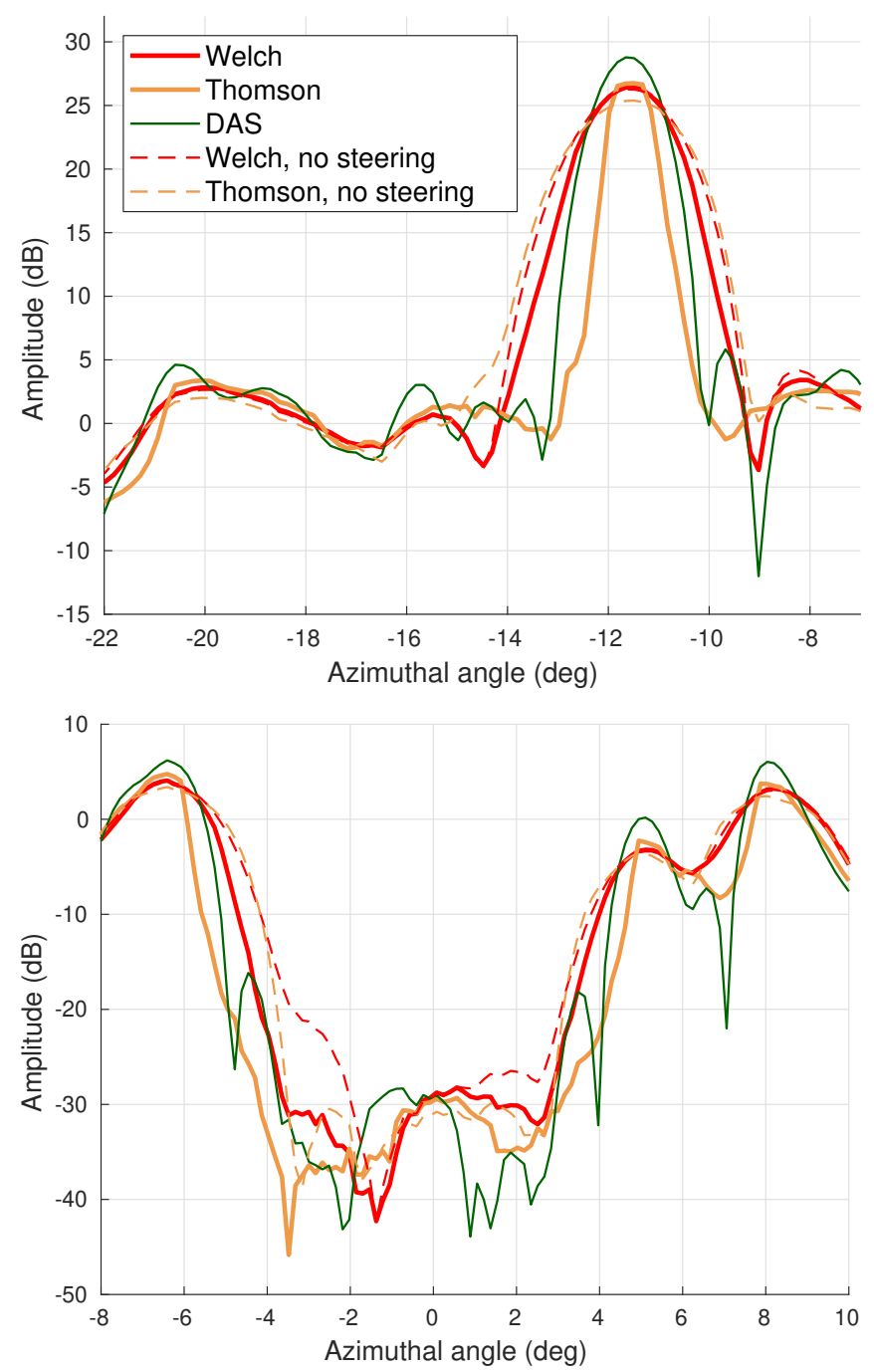

Figure 14. Azimuthal image cuts from the recorded phantom data. The locations are indicated in Figure 13a). a) and b) correspond to the cuts at ranges $83 \mathrm{~mm}$ and $60 \mathrm{~mm}$, respectively.

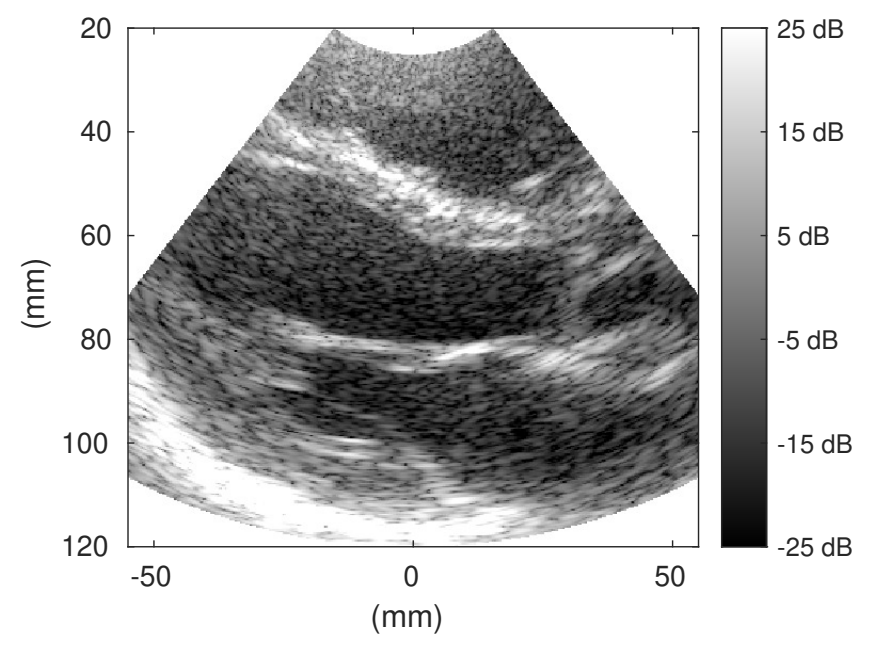

(a) DAS

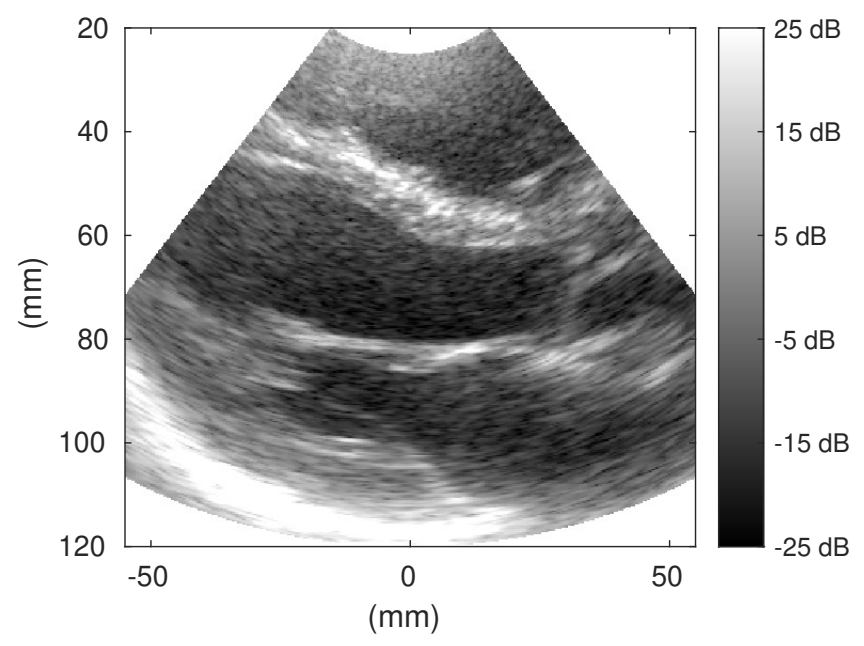

(b) Thomson

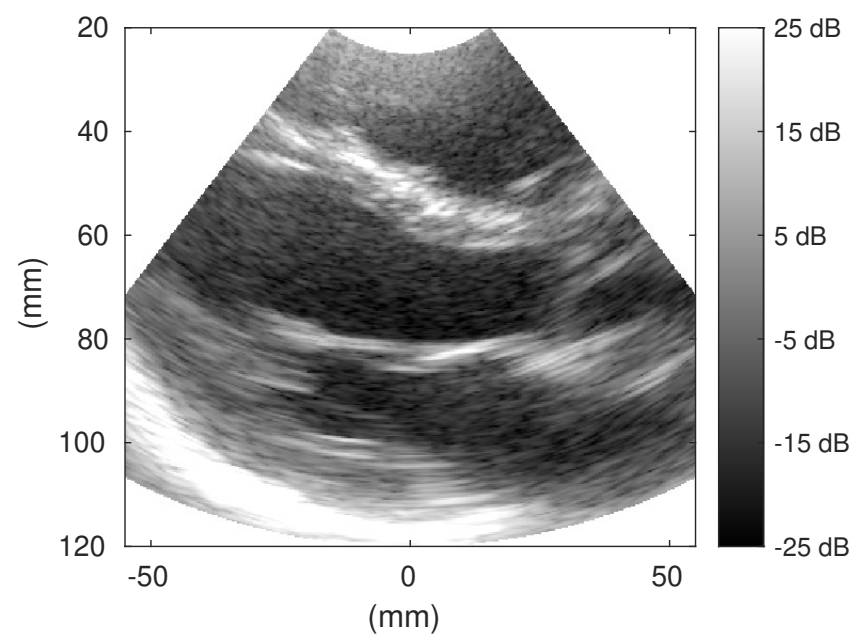

(c) Thomson, no steering

Figure 15. In-vivo data. a) A DAS-processed image, b) and c) steered and unsteered receive-side compounded versions of a), respectively. 